\title{
When Should We Perform Endoscopy for Patients with Upper Gastrointestinal Bleeding?
}

\author{
Kyoungwon Jung and Moo In Park \\ Department of Internal Medicine, Kosin University College of Medicine, Busan, Korea
}

See "Endoscopy Timing in Patients with Acute Upper Gastrointestinal Bleeding” by Gonçalo Alexandrino, Tiago Dias Domingues, Rita Carvalho, et al., on page 47-52.

Acute upper gastrointestinal bleeding is an important emergency condition, and endoscopic intervention plays a pivotal role in its management. ${ }^{1}$ Although there have been technical advances in the field of therapeutic endoscopy, the management of gastrointestinal bleeding remains challenging. ${ }^{2}$ In addition, the increasing use of antiplatelet agents and anticoagulants has further complicated clinical decision making. ${ }^{3}$ The optimal timing of endoscopy in patients with upper gastrointestinal bleeding remains controversial. ${ }^{4}$ Current guidelines recommend performing endoscopic examination within $24 \mathrm{~h}$ of the onset of bleeding. ${ }^{2,5,6}$ In contrast, urgent endoscopy within $12 \mathrm{~h}$ is recommended when variceal bleeding is suspected. $^{7}$

In a recent review article, the authors compared the clinical outcomes of endoscopy within $12 \mathrm{~h}$ (urgent or very early endoscopy) and endoscopy within $24 \mathrm{~h}$ for nonvariceal upper gastrointestinal bleeding. It was found that urgent endoscopy increased the utility of endoscopic treatment; however, the overall outcomes, including mortality, recurrent bleeding, or the need for surgical intervention, did not differ between the 2 groups. These results led to the conclusion that urgent endos-

Received: December 6, 2018 Revised: December 21, 2018

Accepted: December 22, 2018

Correspondence: Moo In Park

Department of Internal Medicine, Kosin University College of Medicine, 262 Gamcheon-ro, Seo-gu, Busan 49267, Korea

Tel: +82-51-990-5205, Fax: +82-51-990-5055, E-mail: mipark@kosinmed.or.kr ORCID: https://orcid.org/0000-0003-2071-6957

(c) This is an Open Access article distributed under the terms of the Creative Commons Attribution Non-Commercial License (http://creativecommons.org/ licenses/by-nc/3.0) which permits unrestricted non-commercial use, distribution, and reproduction in any medium, provided the original work is properly cited. copy should be considered only in selected patients at a high risk. ${ }^{4}$ Another retrospective study divided patients into lowrisk and high-risk groups according to the Glasgow-Blatchford score (GBS), and showed that urgent endoscopy was associated with a more than 5 -fold increase in the risk of worse outcomes, including rebleeding, the need for intervention, and death, compared with conventional endoscopy. ${ }^{8}$ In a subgroup analysis, similar results were found in the low-risk group, whereas in the high-risk group the timing of endoscopy was not a significant predictive factor of outcomes. On the basis of the results, it seems that urgent endoscopy is not beneficial but harmful in patients with a low risk. However, considering the retrospective nature of this study, it can be speculated that patients who underwent urgent endoscopy may have had a serious clinical manifestation or have been unstable, resulting in earlier endoscopic examination, and therefore were associated with a worse outcome than those with a stable condition. For this reason, urgent endoscopy might be associated with a worse outcome although the GBS suggests a low risk. That is, the timing of endoscopy reflects the severity and urgent endoscopy itself might not be associated with a worse outcome.

In this issue of Clinical Endoscopy, Alexandrino et al. ${ }^{9}$ investigated the relationship between the timing of endoscopy and the clinical outcomes in patients with upper gastrointestinal bleeding, including both variceal and nonvariceal bleeding. Patients were classified into the low- and high-risk groups based on the GBS. Comparisons were made with respect to the cause of gastrointestinal bleeding and the timing of endoscopy: variceal versus nonvariceal bleeding and very early endoscopy (within $12 \mathrm{~h}$ ) versus early endoscopy (within 12-24 
h). The primary composite outcome was defined by the sum of each event, including rebleeding, the need for surgery or intensive care unit care, or death, during the hospital stay. In this study, the risk of a composite outcome was $73.9 \%$ lower in the early endoscopy group than in the very early endoscopy group (odds ratio, 0.261; 95\% confidence interval, 0.113-0.602; $p=0.001$ ). Subgroup analysis showed that very early endoscopy was associated with a poor outcome in patients with a low risk and in those with nonvariceal bleeding. These results are consistent with a previous study showing that patients with a low initial GBS and hemodynamic stability could not be properly managed before endoscopy. As for patients with hemodynamic instability, initial resuscitation and stabilization are more effective than very early endoscopy in preventing unfavorable outcomes. Indeed, the time to endoscopy was not a significant predictor of composite outcome in patients with a high risk or those with variceal bleeding in stratified analysis.

In conclusion, this study confirmed that very early endoscopy for upper gastrointestinal bleeding is associated with worse clinical outcomes. It seems that very early endoscopy is not necessary for all patients with upper gastrointestinal bleeding. Rather, very early endoscopy may be beneficial for patients after adequate pre-endoscopy management, including resuscitation, blood transfusion, and use of a proton pump inhibitor. However, this study is also a retrospective analysis and the timing of endoscopy was left to the discretion of the treating physician. Well-designed prospective studies are needed to clarify the benefit of urgent or very early endoscopy for patients with acute upper gastrointestinal bleeding.

Conflicts of Interest

The authors have no financial conflicts of interest.

\section{REFERENCES}

1. Tielleman T, Bujanda D, Cryer B. Epidemiology and risk factors for upper gastrointestinal bleeding. Gastrointest Endosc Clin N Am 2015;25:415-428.

2. Karstensen JG, Ebigbo A, Aabakken L, et al. Nonvariceal upper gastrointestinal hemorrhage: European Society of Gastrointestinal Endoscopy (ESGE) cascade guideline. Endosc Int Open 2018;6:E1256-E1263.

3. Cai JX, Saltzman JR. Initial assessment, risk stratification, and early management of acute nonvariceal upper gastrointestinal hemorrhage. Gastrointest Endosc Clin N Am 2018;28:261-275.

4. Kumar NL, Travis AC, Saltzman JR. Initial management and timing of endoscopy in nonvariceal upper GI bleeding. Gastrointest Endosc 2016;84:10-17.

5. Hwang JH, Fisher DA, Ben-Menachem T, et al. The role of endoscopy in the management of acute non-variceal upper GI bleeding. Gastrointest Endosc 2012;75:1132-1138.

6. Laine L, Jensen DM. Management of patients with ulcer bleeding. Am J Gastroenterol 2012;107:345-360; quiz 361.

7. Hwang JH, Shergill AK, Acosta RD, et al. The role of endoscopy in the management of variceal hemorrhage. Gastrointest Endosc 2014;80:221227.

8. Kumar NL, Cohen AJ, Nayor J, Claggett BL, Saltzman JR. Timing of upper endoscopy influences outcomes in patients with acute nonvariceal upper GI bleeding. Gastrointest Endosc 2017;85:945-952.e1.

9. Alexandrino G, Domingues TD, Carvalho R, Costa MN, Lourenco LC, Reis J. Endoscopy timing in patients with acute upper gastrointestinal bleeding. Clin Endosc 2019;52:47-52. 